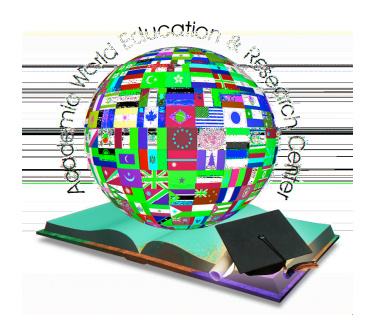

G J $\quad$ B

- 1 Global Journal of

M

।

www.awer-center/gjbem

\title{
Assessment of returns from Zero impact of strategic strategy on firm's performance
}
Solomon Arhin *, B
A $\quad G$
M
D B A M
C

Suggested Citation:

A A

Global Journal of Business, Economics and Management 5
$\mathrm{N}$
D
P D A I IACOB B
A

$E$
P OCL D A
E
C A

\section{Abstract}

C

OA OE M $\quad$ G

IBM P

B

K

OA

OE G 
A

A

Economics and Management 5

Global Journal of Business,

\section{Introduction}

1.1 Research Backgrounds and Motives

B

C

1.2 Statement of the problem

A

C

1.4 Research Questions and Hypothesis

$\mathrm{H}$

A

K K

1.6. Organization of the study and Research procedures:

I C II

C III

C

1.7. Significance of the study

F

E 

Economics and Management 5

1.8. Limitations and Delimitations

A $\quad F$

\section{Literature Review}

E

F

D

A $\quad \mathrm{H}$

0

A

G

J $\quad \mathrm{H}$

$\mathrm{H}$

A

F

A

$\begin{array}{ll}\mathrm{G} & \mathrm{F} \\ \mathrm{K} & \mathrm{N} \\ & \mathrm{F}\end{array}$

N
$\mathrm{F}$

G

$\begin{array}{ll}\text { K } & \text { J } \\ M & P\end{array}$

H

N

B $\quad M$

A

$\mathrm{H}$

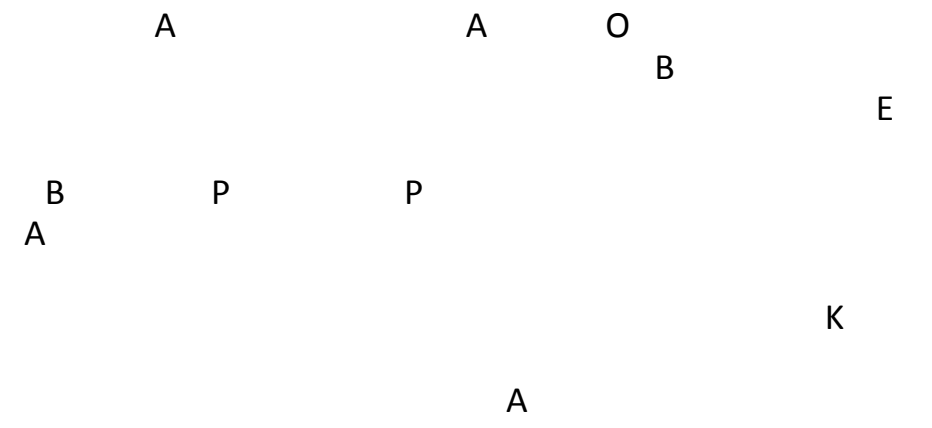


Economics and Management 5

I

C

L

L C

O

P $\quad K$

I

M 
A

Economics and Management 5

3. Research Methodology

3.1 Research Design and Approach

A

$\mathrm{H}$

Q

I

3.2 Sampling

M

A

\subsection{Assumptions}

A

A

A

A

3.4 Research Instrumentation
IBM P 
A

A

Economics and Management 5

Global Journal of Business,

OA

A OE M E I

3.5 Data Collection Procedure

B

$P \quad P$

A

C

$E$

C

$\begin{array}{llll}E P & P E & A \\ G & M & M\end{array}$

3.6 Validity

L $\quad O$

E

\section{Results of Study}

4.1 Data Analysis and Statistical Analysis Tool IBM P

IBM P

$P$

\begin{tabular}{|c|c|c|c|c|}
\hline & & $O A$ & $\mathrm{OE}$ & PP \\
\hline$N$ & $M$ & & & \\
\hline$M$ & & & & \\
\hline $\mathrm{M}$ & & & & \\
\hline
\end{tabular}

A 
A

A

Global Journal of Business,

Economics and Management 5

\begin{tabular}{cc}
$F$ & \\
\hline$P P$ & $M$
\end{tabular}

M

M

M

H

I

L

IBM P

F

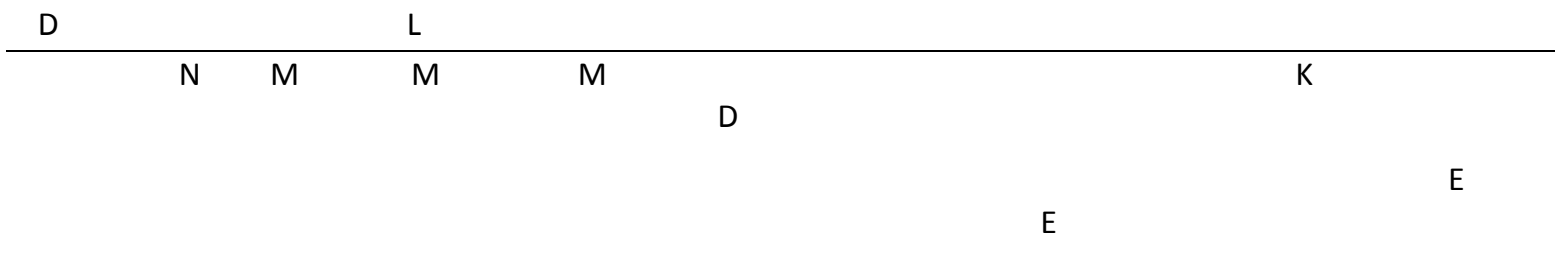

$\mathrm{OA}$

PP

OE

M

N 
Economics and Management 5

\begin{tabular}{|c|c|c|c|c|c|c|}
\hline & & & $\mathrm{OA}$ & $\mathrm{OE}$ & PP & $M$ \\
\hline & $P$ & $C$ & & & & \\
\hline \multirow[t]{2}{*}{$O A$} & $\mathrm{~N}$ & & & & & \\
\hline & $P$ & $C$ & & & & \\
\hline \multirow[t]{3}{*}{$\mathrm{OE}$} & & & & & & \\
\hline & $\mathrm{N}$ & & & & & \\
\hline & $P$ & C & & & & \\
\hline \multirow[t]{3}{*}{ PP } & & & & & & \\
\hline & $\mathrm{N}$ & & & & & \\
\hline & $P$ & C & & & & \\
\hline \multirow[t]{5}{*}{$M$} & & & & & & \\
\hline & $\mathrm{N}$ & & & & & \\
\hline & $P$ & $C$ & & & & \\
\hline & $\mathrm{N}$ & & & & & \\
\hline & $\mathrm{N}$ & & & & & \\
\hline
\end{tabular}

$\mathrm{H}$

H A

\section{OA G M}

\begin{tabular}{llllll} 
& \multicolumn{3}{c}{ F } & & \\
& $\mathrm{D}$ & & $\mathrm{OA}$ & \\
\hline & $\mathrm{N}$ & $\mathrm{M}$ & $\mathrm{M}$ & $\mathrm{M}$ & $\mathrm{D}$ \\
\hline $\mathrm{N}$ & & & & & \\
\hline
\end{tabular}

IBM P

OA

G

\begin{tabular}{lllllll}
\hline PP & N & $M$ & D & E & $M$
\end{tabular}

OA 

A

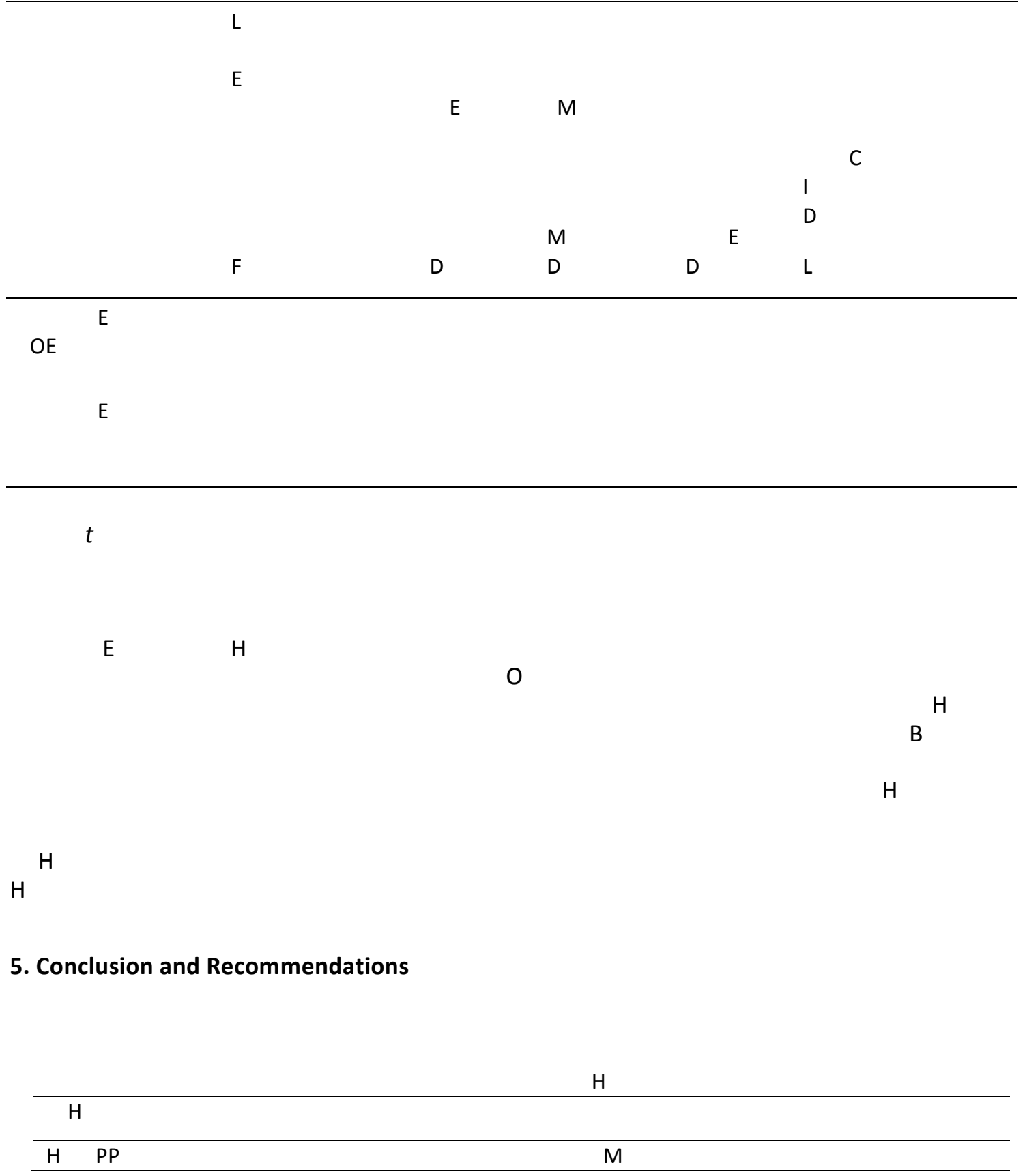




\section{Recommendation for further study \\ $\mathrm{F}$}

E $\quad A$

\section{References}

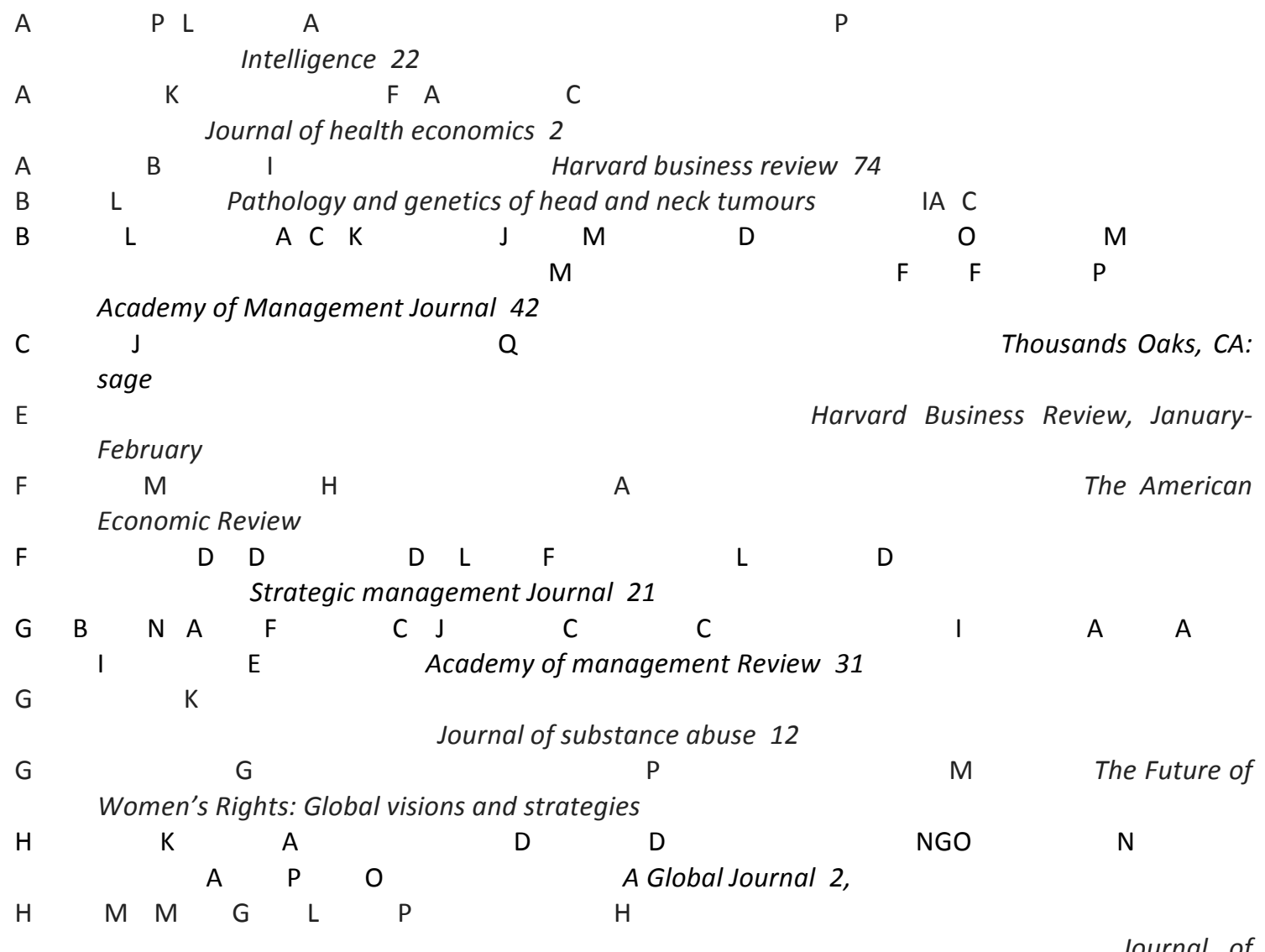

Operations Management 24 
Economics and Management 5

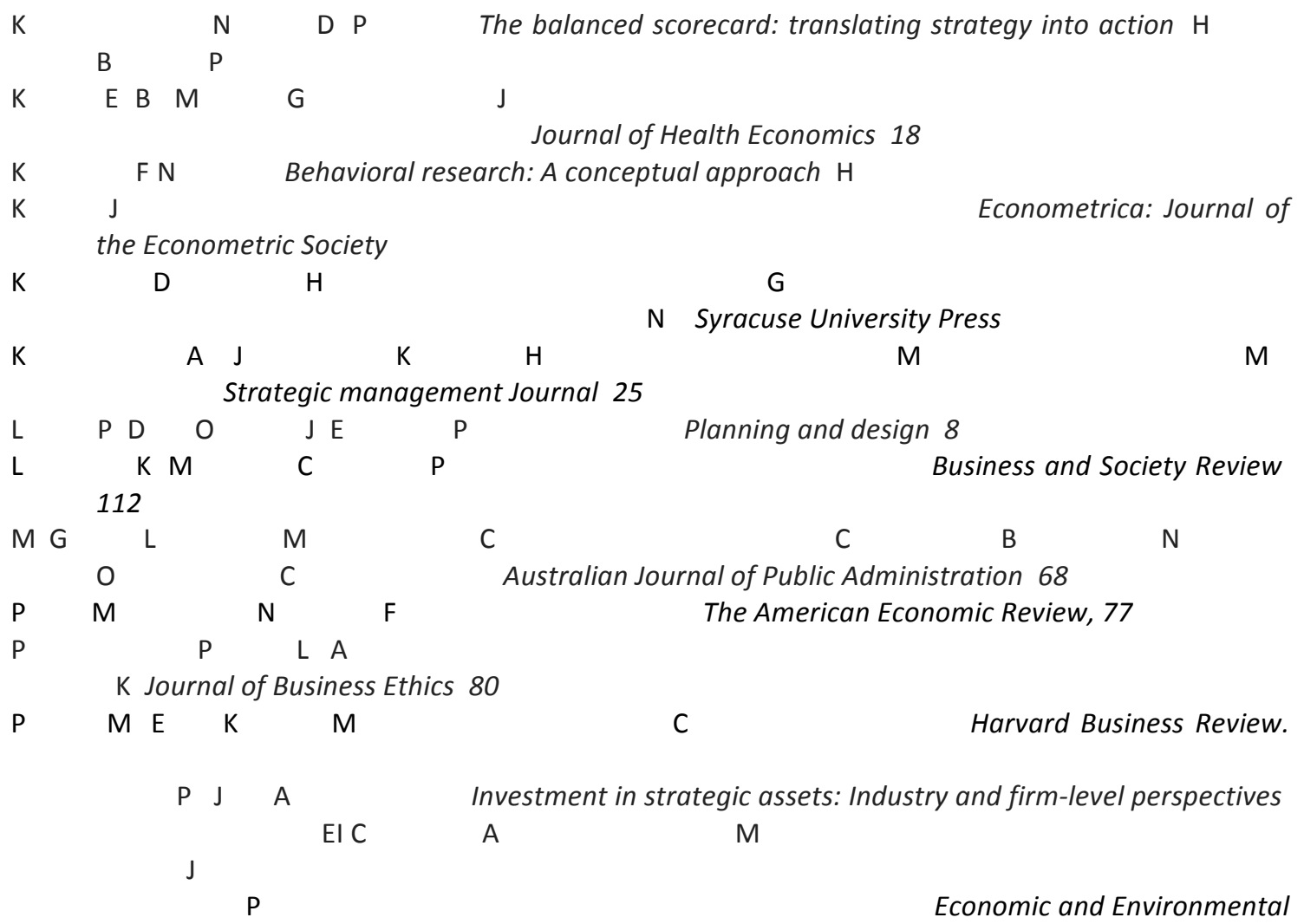
studies 11

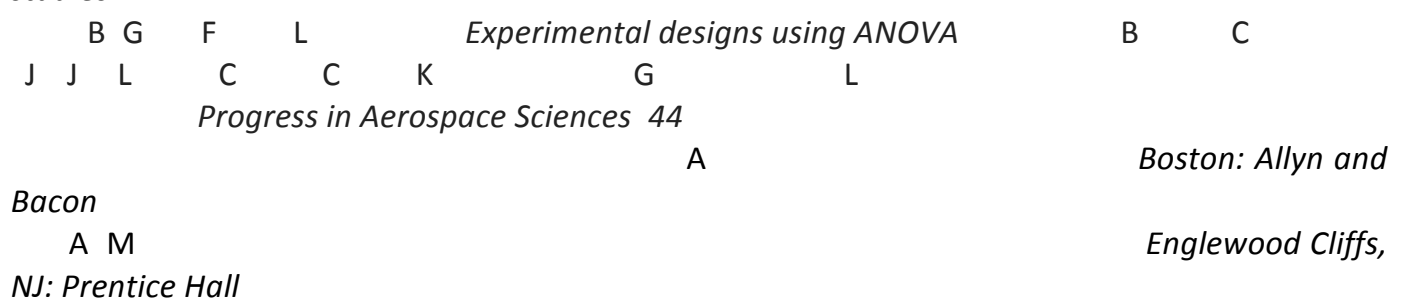

